\title{
DE LILIPUT A BROBDINGNAG: NOTA SOBRE LAS RELACIONES MICRO-MACRO EN SOCIOLOGIA
}

\author{
José Enrique Rodríguez Ibáñez \\ Universidad Complutense
}

\begin{abstract}
RESUMEN
Este trabajo analiza el problema de las relaciones micro-macro en Sociología desde una perspectiva gradacional que trata de solventar el dualismo en el enfoque de la cuestión, tanto en el campo de la teoría como en el de los programas de investigación. Para ello se remite a la tradición de la teoría sociológica y se detiene en particular en aportaciones cognitivistas y emotivistas al estudio de los microfundamentos del orden social (Cicourel, Collins, Scheff).
\end{abstract}

\section{GENERALIDADES INTRODUCTORIAS}

Como Swift (y Gulliver, su criatura), son muchos los sociólogos que estiman que las parcelas de la sociedad pueden ubicarse en dos países diferentes: el de los «enanos» (donde la pequeñez de las unidades hace evidente y transparente el cúmulo de problemas ante los ojos del engrandecido observador) y el de los «gigantes» (donde, por el contrario, el tamaño descomunal de los partícipes relativiza la perspectiva y obliga a aventurar hipótesis explicativas abiertas). Ni que decir tiene que "Liliput» sería el mundo de lo «micro» y «Brobdingnag» el mundo de lo "macro». Pero esta perezosa segregación de la realidad y los modelos de acceso a ella no sólo está sesgada de origen, sino que, además, es contraproducente. Está sesgada porque otorga transparencia u oscuridad a los ámbitos de la sociedad según su inmediatez (lo cual es gratuito: hay micro- 
escenarios impenetrables y macroescenarios diáfanos). $\mathrm{Y}$ es contraproducente porque rompe con el continuum individuo-sociedad en vez de partir de él, como parece razonable si se quiere elaborar un buen modelo teórico sociológico.

Con esto, lógicamente, no pretendo decir que no sea sensato hablar de relaciones micro-macro; sólo quiero dar por sentado que esas relaciones deben ser de continuidad y no de enfrentamiento o exclusión mutua. En realidad, las grandes propuestas de la Sociología no han hecho otra cosa que tratar de explicar tal continuum, eso sí desde distintos puntos de vista epistemológico-metodológicos y/o ontológico-descriptivos. Desde este último punto de vista -el ontológico-, la realidad social puede concebirse como una superposición de individuos, como un organismo fruto de la superposición anterior (pero superior y distinto a ella en su conjunto) o como una estructura normativa que hace de los individuos meros agentes o portadores de la megarracionalidad institucional. La primera postura — «interindividualista»— sería enteramente «micro»; la segunda — «organicista»— sería «micro-macro», y la tercera — «colectivista»— sería abiertamente «macro».

Pasemos ahora al enfoque epistemológico-metodológico. Aquí caben dos ángulos alternativos: el «individualista» (que reconstruye el orden social a partir de la perspectiva del actor intencional) y el "holista» (que reconstruye el orden social a partir de la realidad ya constituida o preexistente al análisis sociológico). La perspectiva «individualista-metodológica», claro está, se reputa "micro-macro», mientras que la "holista» se reputa "macro-micro».

Si cruzamos las dos perspectivas, ontológica y metodológica, obtenemos seis celdillas en las que, siempre al riesgo de la simplificación, estaremos en condiciones de ubicar a algunas de las más importantes propuestas de la teoría sociológica clásica y contemporánea. He aquí el cuadro que paso inmediatamente a comentar:

\begin{tabular}{|c|c|c|c|}
\hline Perspectiva ontológica & Interindividualismo & Organicismo & Colectivismo \\
\hline 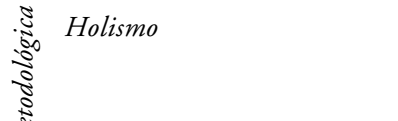 & $\begin{array}{c}\text { Freud } \\
\text { Estructural- } \\
\text { funcionalismo }\end{array}$ & $\begin{array}{l}\text { Durkheim } \\
\text { Luhmann }\end{array}$ & Marx \\
\hline 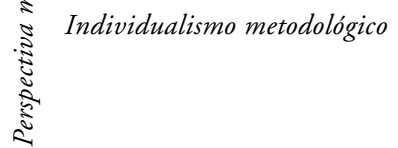 & $\begin{array}{l}\text { Simmel } \\
\text { Interaccionismo } \\
\text { simbólico } \\
\text { Etnometodología }\end{array}$ & $\begin{array}{c}\text { Max Weber } \\
\text { Habermas }\end{array}$ & $\begin{array}{l}\text { Pareto } \\
\text { Elección racional } \\
\text { Giddens }\end{array}$ \\
\hline
\end{tabular}

Insistiendo una vez más en que estas clasificaciones son siempre aproximadas y no deben ser tomadas como una verdad matemática, me atrevo a defender la categorización propuesta, que desarrollo de izquierda a derecha del cuadro, empezando por la fila superior. 
La teoría estructural-funcional, en primer lugar, aun cuando parta hipotéticamente «de la perspectiva del actor» (ésta fue siempre la obsesión de Parsons), en realidad termina dando primacía explicativa a las macrofunciones del sistema global, las cuales gravitarían sobre el actor individual. Como su precedente freudiano - que explica al yo en función del superyo-, el estructuralfuncionalismo clásico combina una ontología interindividual con una aproximación holista a la misma.

Por lo que se refiere a la «realidad sui generis» de Durkheim o a los «sistemas» de Luhmann, no creo resulte problemático admitir que ambos aceptan una visión metaindividual u orgánica de la sociedad cuya vía de acceso metodológico debe ser forzosamente holista para no incurrir en supuesta gratuidad subjetivista y acientífica.

En cuanto a Marx, sería ocioso insistir en su cualidad de representante por excelencia de una teoría holista que configura a la realidad como suma de estructuras.

Entrando en los representantes del individualismo metodológico, nos topamos con un gran clásico — Simmel_ y algunas direcciones — como el interaccionismo simbólico y la etnometodología- que desean reconstruir las reglas formales (y poco explícitas) del orden social a partir de las redes interactivas de los usos cotidianos. El tejido interindividual está presente en los dos órdenes, descriptivo y explicativo.

En lo relativo al campeón del individualismo metodológico - Max Weber - y al teórico de la acción comunicativa y la razón discursiva - Habermas-, ambos conciliarían una visión intersubjetiva de las raíces de la sociedad con un designio "organicista» de la realidad social.

Por fin, las teorías del rational choice (inspiradas en mucha medida por Pareto) y la teoría de la "estructuración» de Giddens compartirían la vocación ontológica estructural con la insistencia en la reconstrucción «individualista metodológica» de la estructura global (sin que, por supuesto, ambas aproximaciones hagan ni digan exactamente lo mismo).

Todos estos enfoques, como antes veíamos, son bien «macro-micro» (celdillas primera y segunda), bien «macro-macro» (tercera celdilla), bien «micromicro" (celdilla cuarta), o bien "micro-macro» (quinta y sexta celdillas). Ninguno desdeña el hecho de que las relaciones interpersonales y las relaciones institucionales o estructurales forman un par explicativo y/o descriptivo útil (que se plasmaría canónicamente, añado yo, en la pareja Gemeinschaft-Gesellschaft de Tönnies); pero sí que difieren en cuanto al grado de primacía de uno de los elementos del par sobre el otro o en cuanto al punto de partida «micro» o "macro» de las hipótesis explicativas. En este sentido, la perspectiva «micro-micro» explora los microfundamentos cognitivos y normativos de un orden "macro» que se da por sobreentendido, mientras que la perspectiva "macro-macro" explora las relaciones de subordinación interestructurales, dando por sobreentendida la génesis intersubjetiva de las mismas. Por su parte, las perspectivas «macro-micro» y «micro-macro» tratan de explicar el conjunto de la realidad a 
la luz de la permanente articulación de esos planos, y a partir de posturas que explican a los átomos o unidades hipotéticas del sistema social desde el todo a la parte («macro-micro») o desde la parte al todo («micro-macro»).

Hoy en día, superada la etapa de considerable confusión que caracterizó a la teoría sociológica desde finales de los años sesenta hasta finales de los ochenta, pudiéramos decir que la toma de partido por lo micro o lo macro como opciones excluyentes y aun beligerantes está fuera de lugar, habiendo consenso en torno a la idea de que lo decisivo no es confrontar a lo "micro" con lo «macro", o viceversa, sino estudiar la gradación analítica y correspondencia empírica que se daría entre ambos planos. De esta manera, los clásicos dejan de ser un arma arrojadiza, el debate entre escuelas contemporáneas es más fecundo y las posibilidades de investigación más omnicomprensivas (Alexander y Giesen, 1987; Gerstein, 1987; Lamo de Espinosa y Rodríguez Ibáñez, 1993; Münch, 1987; Rodríguez Ibáñez, 1992)ํ․

${ }^{1}$ El libro colectivo en el que se encuentran las aportaciones de Alexander y Giesen, Gerstein y Münch recién mencionadas (The micro-macro link, publicado por la Universidad de California y compilado por Jeffrey Alexander, Bernhard Giesen, Richard Münch y Neil Smelser) constituye un sólido "estado del arte» de la problemática aquí tratada. Resaltaré brevemente los contenidos de la introducción, a cargo de Alexander y Giesen, y del trabajo de Münch.

Alexander y Giesen realizan una lectura de la tradición sociológica a la luz de las relaciones micro-macro, estimando que Marx y Durkheim son los primeros grandes sintetizadores de la perspectiva macro, siéndolo Mead de la perspectiva micro (los tres de una manera matizada y no unilateral, por supuesto). La primera gran síntesis completa entre lo micro y lo macro vendría de la mano de Max Weber (quien fundió intencionalidad y racionalidad), alcanzando con posterioridad mayor capacidad de síntesis la teoría del sistema general de la acción de Parsons.

También a la sombra de Parsons, Münch describe en el terreno de lo empírico cuál es la «interpenetración de la microinteracción y las macroestructuras en un orden complejo y contingente». Para ello utiliza la tetralogía parsoniana AGIL, dispuesta en un eje de coordenadas que representan dos órdenes gradacionales: el de la complejidad simbólica de las relaciones sociales y el de la normatividad de la acción social. La sinuosidad del tejido social podría, así pues, quedar configurada como un flujo a cuatro bandas entre un subsistema de intercambios con alto nivel de regulación tanto simbólica como normativa (subsistema A); un subsistema de toma de decisiones de alto nivel simbólico y baja normatividad (subsistema G); un subsistema comunitario de baja complejidad simbólica y también baja normatividad (subsistema I), y un subsistema de discurso racional dotado de alta normatividad y baja densidad simbólica (subsistema L). Traduciendo a román paladino las cuatro esquinas de Münch, podríamos pensar en las siguientes subáreas del orden social: relaciones económicas (A), relaciones políticas $(G)$, relaciones intersubjetivas (I) y relaciones de comunicación pública o relativa a la transmisión del conocimiento (L). Nótese que Münch no se refiere a un único ángulo institucional o macro, sino a cuatro vertientes en las que lo micro y lo macro se simultanean. Gráficamente, el esquema del autor es como sigue:

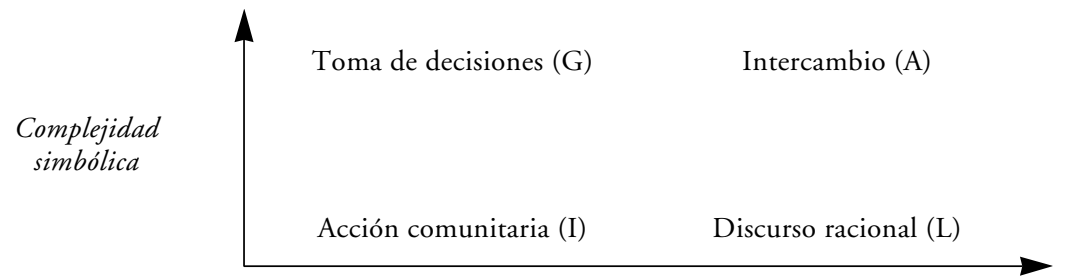

Normatividad de la acción 
No quisiera, empero, dar por zanjada la cuestión. Sí que me parecería redundante abundar sobre problemas y soluciones que la literatura citada ha tratado ya con suficiente extensión. En cambio, no me lo parece, y a ello voy a dedicar el próximo epígrafe, detenerme en aportaciones más o menos recientes que tratan de profundizar en la vía «micro-micro», con la ambición de sentar, con todas sus consecuencias, los microfundamentos del orden social. Mencionaré tres tipos de indagación en torno a los «microfundamentos», una de índole cognitivista (Cicourel y D’Andrade) y otras dos de índole emocional (Collins y Scheff).

\section{EN BUSCA DE LOS «MICROFUNDAMENTOS» DEL ORDEN SOCIAL}

Quiero destacar la radicalización "micro» del asunto que nos ocupa, a cargo de autores contemporáneos, porque constituye una suma de aportaciones muy ambiciosas y no demasiado conocidas que, sin ser definitivas, como veremos, poseen una innegable plausibilidad como modelo teórico y programa de investigación. Existen otras plausibilidades, propias de modelos y programas concurrentes, que daré por conocidas y discutiré in genere en la conclusión, de modo similar a como han sido discutidas las grandes escuelas y tradiciones sociológicas en la introducción.

Empecemos por la obra de Aaron Cicourel. Este autor ha evolucionado de la etnometodología garfinkeliana a una antroposociología de orientación cognitivista. Cicourel, en concreto, acepta en su fase madura un marco epistemológico coincidente con el tipo de ciencia cognitiva propugnada por Rumelhart. No es éste el lugar apropiado para extenderse en polémicas especializadas de la ciencia cognitiva. Diré, resumidamente, que la postura defendida por Rumelhart entiende a la cognición como un proceso emergente de adquisición de información y archivado neuronal que se diversifica en contenidos particulares funcionalmente interrelacionados. La cognición sería una gran traslación neurofisiológica del conjunto de las percepciones del sujeto, las cuales configurarían una suma cada vez más compleja de códigos y lenguajes. Este cognitivismo, de orientación neurofisiológica y computacional, difiere del cognitivismo de Francisco Varela, para el que la situación concreta en que tienen lugar los procesos de cognición crea la configuración psicofísica definitiva del sujeto (Rumelhart y MacLelland, 1986; Varela, 1992).

Pues bien, puesto a afirmarse en la epistemología cognitivista, como manto de amparo teórico que le salve del relativismo de la etnometodología clásica de Garfinkel, Cicourel opta por Rumelhart, y no por un situacionismo filosóficamente afín a la fenomenología como el de Varela, que posiblemente le retrotraería al punto de partida.

El estudio pormenorizado de los mecanismos elementales de la cognición suministra a nuestro autor una base fundante para su propuesta en torno a la naturaleza del orden social. Según Cicourel, este último orden no es sino la 
versión ampliada de aquello que cada individuo — cada cerebro- realiza infinidad de veces al día, esto es, la ordenación de los dictados de la cognición con arreglo a un sistema de categorías cuya expresión última la forman la sintaxis y los lenguajes verbales. De esta manera los lenguajes permiten establecer análisis comparativos, a partir de la mayor relevancia de unas unidades sintácticas y/o semánticas frente a otras, en cada uno de ellos. Estaríamos en presencia del tipo de Antropología cognitiva llevada a cabo por Roy D'Andrade². Cicourel da un paso más y se acerca a la utilización de los modelos cognitivo-sociolingüísticos en el estudio de la estructura social. El autor, en concreto, opta por entender que el orden institucional o burocrático "macro» —el que se escenifica, por ejemplo, en hospitales, dependencias gubernamentales o aulas- es también una suma de lenguajes prescriptivos que anclan su sistema de reparto de prescripciones, prohibiciones y codificaciones en una mentalidad colectiva traducida a discursos y prácticas. Así, pues, el viejo sueño de Garfinkel de inferir la negociación del orden social a partir de las prácticas de la vida cotidiana halla en Cicourel un referente sólido que remite esas prácticas a una suerte de "gramática de la cognición», la cual permite desbrozar los actos del comportamiento interactivo, así como su traducción verbal y la proyección de la misma a los órdenes discursivos institucionales (Cicourel, 1975, 1978, 1980, 1981).

Nos encontramos ante un modelo que da mucho juego para el análisis sociolingüístico micro y macro, pero que puede tender a identificar en demasía realidad social con discurso, dejando fuera a aquellos aspectos más imprevistos o irrepetibles del registro humano. Quizá por esto mismo, otros autores prefieren compartir con Cicourel la radicalización de lo micro como vía de acceso a la explicación sociológica, si bien adoptando una concepción preferentemente emocional de la psique de los individuos.

Este es el caso de Randall Collins, quien publicó en la pasada década un texto emblemático al respecto (Collins, 1981). Aquí, el autor se distancia desde un principio de las posturas «micro» de orientación cognitivista o utilitarista/decisionista racional. "La conducta cotidiana no obedece a modelos racionalistas de cognición y toma de decisiones» — señala Collins—. A su juicio, por tanto, «las explicaciones en términos de normas, reglas y asunción de roles (role taking) deben ser abandonadas (...), necesitándose, a cambio, un micromecanismo que esté en condiciones de explicar las acciones repetitivas sobre las que se levanta la estructura social, remitiéndolas a unas bases no cognitivas de la interacción y la percepción» (Collins, 1981: 985).

A tales micromecanismos explicativos los denomina el autor "cadenas rituales de interacción", las cuales crean y transmiten de abajo arriba las principales coordenadas de la sociedad en forma de «símbolos y mitos culturales» y

2 D’Andrade (1981) utiliza el mayor o menor énfasis que las lenguas otorguen a sus diversas categorías para trazar un paralelo con el perfil antropológico de las culturas que les sirven de soporte. Estas categorías verbales (con sus consiguientes correlatos «macro») son las siguientes: A, «afirmaciones»; B, «requerimientos»; C, «preguntas»; D, «reactivos» (acuerdo/desacuerdo); E, «expresivos» (aprobación/desaprobación); F, «putativos» (promesas, ofrecimientos). 
«energías emocionales». Si Collins no tiene empacho en admitir que estas cadenas pueden ser tildadas prima facie de conversaciones, se trataría, sin embargo, de una clase de comunicación no exclusivamente verbal, sino fundamentalmente emotiva. Según expresa Collins, «se trata de energías emocionales transmitidas mediante contagio entre los miembros de un grupo, a través de flujos que operan de modo muy parecido a la serie de negociaciones que generan los precios en el seno de un mercado» (Collins, 1981: 994).

El autor cruza la noción durkheimiana de "ritual» con la concepción dramatúrgica de la interacción de Goffman, para dejar sentada una teoría de la continuidad micro-macro que arranca de la "causalidad microsituacional» y se vuelca en «recursos culturales» y «energías emocionales» que son los que definen las dimensiones estructurales o «macro». La sociedad, en definitiva, es para Collins un flujo y reflujo de identidades, lealtades y significaciones ancladas en buena medida en el basamento emotivo y fabulador de la psique.

La pretensión universalizante de esta postura despertó críticas que achacaban al autor el hecho de que desdeñara en demasía la dimensión de poder y desigualdad económica. A esto Collins respondió de manera pormenorizada, aunque en mi opinión no del todo convincente (Collins, 1987).

Para el autor, en efecto, la posición social y la propiedad son rasgos básicos de la «microsituación estructurada». La primera es fruto de una negociación dramática y eventualmente conflictiva, mientras que la segunda es, además de condición preexistente, el resultado estructural de unas continuidades micromacro presididas por la desigualdad estratificacional. Bien, Collins parece salvaguardar su esquema, pero, al menos para mi gusto, al precio de una petición de principio: el poder y la desigualdad podrían ser rasgos emergentes de las "cadenas de interacción ritual» siempre que se partiera de ellos como algo ya constituido.

Quizá consciente de lo anterior, el autor se atrevió a romper el nudo gordiano de tal petición de principio, reclamando una auténtica revolución filosófica del pensamiento occidental que le permitiera dotar a su teoría micro-emocional de los necesarios requisitos de fundamentación epistemológica (Collins, 1990).

La solución, ahora, es acabar con la noción de "libre albedrío», presente no sólo en la tradición cristiana, sino también en las tradiciones liberal/utilitarista, pactista y hasta organicista/socialista, admitiendo el determinismo que ejerce sobre los procesos de interacción ritual un difuso depósito de «energías emocionales». Estas energías constituirían la materia prima de la sociedad, y sería su escenificación situacional (y ascendente, en términos micro-macro) la que derivaría en la complejización económica, política y cultural. Con todos los respetos, esto, a mí particularmente, me suena a reedición del freudo-marxismo con ribetes interaccionistas, lo cual no zanja polémicas teóricas, sino que las reabre en el punto de partida en que las dejaron clásicos como Freud, Marcuse o Mead.

Si el cognitivismo de Cicourel concluimos que derivaba hacia una confluencia entre los discursos y la realidad social, y, sensu contrario, el conflictualismo 
emotivo de Collins, según acabamos de ver, derivaría hacia una confluencia entre impulsos y sociedad, la propuesta de Scheff (1990) diríase que busca un justo término entre ambos extremos.

Este autor, formado en una tradición interaccionista atenta a campos como la producción social de la enfermedad mental y las relaciones entre iluminación estética y conocimiento sociológico ${ }^{3}$, culmina su carrera en la obra que nos ocupa, dedicada a forjar toda una teoría sociológica de base "micro», centrada en las emociones como referencia primera.

En efecto, Scheff arranca del impulso emotivo como tamizador inicial del curso de la acción y aun del entramado estructural de la misma. Serían, en opinión del autor, sentimientos tales como la vergüenza o el orgullo los que animarían a proceder de una manera u otra a los actores sociales, interponiendo además dichos sentimientos ante los actores un determinado manto perceptivo que haría de la estructura social real algo elusivo o incluso invisible. Pero las emociones, transmutadas en acción —-más o menos consciente, más o menos distorsionada, más o menos aceptada-, generan un orden normativo hecho a partes iguales de discurso y estructura. De ahí que la estructura social deba ser reconstruida a partir de sus componentes "micro", y estos últimos inferidos de las emociones prevalecientes. No obstante, culmina Scheff, la «escalera micromacro» funciona también en dirección "macro-micro», a través de las reacciones emotivas de las psicologías individual y colectiva. Dichas reacciones «ascienden" de lo micro a lo macro o "descienden» de lo macro a lo micro en milésimas de segundo, utilizando por ende una lógica que no es formal o deductiva ni empírico-inductiva, sino «abductiva», al modo pragmatista. Scheff, para estos propósitos, se apoya en precedentes esperables, como el de Goffman, y en otros más insólitos, como el Wright Mills de La imaginación sociológica (autor este último partidario, como se sabe, de ir de la intuición personal a la apreciación genérica, en un contexto de investigación «artesanal»).

Scheff quiere poner a prueba este su particular prisma, comparándolo con el método de Durkheim en relación con el estudio del suicidio. Quedarse a

3 Aunque no exceda de lo anecdótico, no quiero dejar de mencionar una curiosa «justa» que protagonizaron Thomas Scheff y Otis Dudley Duncan en el verano de 1985 y que tuve la ocasión de presenciar. El escenario era el Departamento de Sociología de la Universidad de California en Santa Bárbara, y el objeto de debate, «las relaciones entre Literatura y Sociología». Scheff —que, aparte de su actividad conocida, inició sus pasos académicos en las ciencias físicas - sostenía que la Literatura, incluida la poesía, es la fuente de la que debe emanar la concreción de hipótesis sociológicas (quedando para la metodología investigadora de inspiración popperiana el resto del trabajo). Por el contrario, Duncan — quien, aparte de ser el reputado coautor de The American occupational structure y el campeón de los modelos longitudinales, es un amante de la civilización grecolatina, un lector empedernido de textos originales clásicos de tal civilización y un músico amateur - mantuvo la tesis de que la investigación social recorre un camino separado de la creación literaria y que ambos caminos no deben mezclarse, por su propio bien. Según concluyó gráficamente, la poesía es demasiado importante como para ponerla en manos de sociólogos. Me temo que fue Duncan el que salió victorioso del torneo floral. 
este respecto con las solas correlaciones estadísticas durkheimianas, arguye Scheff, puede hacer perder de vista la motivación más profunda del acto suicida, perdiéndose no sólo pathos en la explicación de tal conducta, sino incluso también valor sociológico causal. En concreto, el autor propone detenerse en el héroe de Goethe, el joven Werther, muerto, sí, por despecho amoroso y prefigurador del romanticismo, pero movido por unas razones que no termina de atrapar sociológicamente la explicación de "suicidio egoísta» que Durkheim hubiera sugerido seguramente. A cambio, Scheff resalta la afrenta que Werther sufre al ser animado a abandonar una reunión de nobles por no pertenecer a tal clase. Sería esta "vergüenza» o ultraje el que disuade a Werther de proseguir sus acercamientos amorosos, cerrándose por añadidura a sí mismo el camino del ascenso social. Es, pues, un sentimiento el que desencadena la construcción y estructuración de la realidad, así como su resultante suicida. Microacción emotivamente fundamentada, discurso y estructura social aparecen forjados en un gradiente bidireccional.

La postura de Scheff es más ecléctica que las de Cicourel y Collins, pues concibe a la realidad como una triple articulación de acciones, discursos y estructuras. Sólo el empeño último en fundamentar todo ello en una sociología de las emociones le distancia de otros modelos racionalistas causal-intencionales, como el de la decisión o elección racional, que considero más sólidos y deberían ser tratados en esta sección, si no fuera porque ya dediqué a ellos todo un trabajo (Rodríguez Ibáñez, 1991). Será basándome en las conclusiones de mi últimamente mencionado escrito como recapitularé acerca de lo debatido hasta ahora, propugnando una actitud no excluyente, sino "transversal», para el enfoque de las relaciones «micro-macro» en sociología.

\section{DE LA DUALIDAD A LA TRANSVERSALIDAD}

El trabajo propio que acabo de mencionar concluía apelando a la aceptación de tres grandes planos de la realidad: un sistema socioeconómico de intercambios generalizados, un sistema político-cultural y un sistema ecobiológico, dotados cada uno de ellos de disciplinas explicativas ad hoc. Esta afirmación salía al paso del «imperialismo microeconómico» en las hipótesis de teoría sociológica, apostando por una gradación — que no eclecticismo- entre los enfoques, cuya última prueba de validez sería justamente el umbral de plausibilidad investigadora desplegada por todos y cada uno de tales enfoques. Más recientemente (Rodríguez Ibáñez, 1997), he tenido ocasión de sintetizar y "desmenuzar» analíticamente a las que considero las «cuatro avenidas fuertes» de la teoría sociológica contemporánea, a saber, la teoría comunicativa, la teoría de sistemas, la teoría de la decisión o elección racional y la teoría cognitivista. No voy a repetir lo expresado en aquellas páginas, pero sí que reiteraré mi criterio de que las opciones teóricas en sociología son «fuertes» cuando logran 
alcanzar universalismo explicativo y cuando su ámbito de aplicación es capaz de saber concatenar lo singular y lo global (o «micro» y «macro»).

Con lo dicho no pretendo llegar a otro término que a la convicción de que las relaciones micro-macro en Sociología no constituyen ni deben constituir una divisoria interteórica (es decir, no deben dividir a las teorías según sus opciones «micro» o «macro»), sino que, por el contrario, son o deben ser un gradiente intrateórico (o sea, una dimensión de concatenación de espacios, contenidos y actores que toda teoría que merezca ser llamada como tal tiene que estar en condiciones de mostrar, dándole la adecuada respuesta en el terreno de las hipótesis, los modelos y las estrategias de investigación).

De esta manera, las relaciones micro-macro dejan de ser una contraposición dual y pasan a ser unos polos hipotéticos de una escala de magnitudes, intencionalidades y consecuencias objetivas de la acción que atraviesa transversalmente todos los órdenes y procesos de la sociedad, así como los resúmenes teóricos de la misma.

En este sentido, Bernhard Giesen (1987) ha elaborado un imaginativo «mapa» al respecto que sienta de manera realista cuáles pudieran ser los itinerarios y jerarquías categoriales y empíricas de tal concatenación transversal.

El autor, en concreto, propone distinguir entre tres realidades: una «simbólica» (concretada en visiones del mundo), otra "práctica» (concretada en instituciones diferenciadas) y otra "material» (centrada en recursos naturales y en la tecnoestructura). Pues bien, el tránsito micro-macro alcanzaría un primer nivel de consolidación en el mundo de las estructuras simbólicas. Estas últimas «invadirían» el mundo de las estructuras prácticas, modificándolas eventualmente. A su vez, el mundo de los recursos naturales bien pudiera incidir, a todos los niveles, sobre las instituciones, las cuales, a su vez, influirían sobre las estructuras simbólicas en la misma medida que ellas mismas son susceptibles de ser influidas por las últimas. Se trata, como se ve, de un círculo de posibilidades e interrelaciones que dinamiza, en lo relativo a la depuración conceptual, el problema que nos ocupa, en forma ligeramente coincidente con la triple distinción de planos que yo antes proponía.

Pero en último extremo lo que importa no es perderse en logomaquias o en preciosismos terminológicos. Lo que importa, como ha señalado Coulter (1996), es saber armonizar la mirada sociológica - la del experto y la del lego- con los resultados generalizables de la misma. Eso es hacer «teoría fuerte», la que permite a los «Gulliveres» que todos y todas somos transitar de «Liliput» a "Brobdingnag» (o viceversa), sin tener que salir de las islas-continente en que moramos. Al final del viaje de la vida y de la muerte que la Sociología se esfuerza en narrar y desvelar, habrá varias versiones en torno al mismo. Las más plausibles de todas ellas no recogerán íntegramente lo narrado y desvelado, pero serán las únicas dignas de competir entre sí, asegurando a teóricos y teorizados una sensación imborrable de continuidad y mutua complementariedad entre la perspectiva y las obras de «enanos» y "gigantes». 


\section{BIBLIOGRAFIA*}

Alexander, J.; Giesen, B.; Münch, R., y Smelser, N. J. (eds.) (1987): The micro-macro link, Berkeley, University of California Press.

AleXANDer, J., y Giesen, B. (1987): «From reduction to linkage», en J. Alexander et al., cit.

Cicourel, A. (1975): «Discourse and text: cognitive and linguistic processes in studies of social structure», Versus, septiembre-diciembre.

- (1978): «Language and society: cognitive, cultural and linguistic aspects of language use», Sozialwissenschaftliche Annalen (Viena), Band 2.

- (1980): «Three models of discourse analysis: the role of social structure», Discourse Processes, 3.

- (1981): "Notes on the integration of micro and macro levels of analysis», en K. Knorr-Cetina y A. Cicourel (eds.): Advances in social theory and methodology. Toward and integration of micro and macro sociologies, Londres, Routledge \& Kegan Paul.

Collins, R. (1981): "On the microfoundations of sociology», American Journal of Sociology, vol. 86, núm. 5 .

- (1987): «Interaction ritual chains, power and property», en J. Alexander et al., cit.

- (1990): "The emotional energy of interaction rituals and the cult of the will», Madrid, XII Congreso Mundial de Sociología.

Coulter, J. (1996): "Human practices and the observability of the "macro-social" ", Zeitschrift für Soziologie, vol. 25, núm. 5 (octubre).

D'ANDrade, R. (1981): "The cultural part of cognition», Cognitive Science, 5.

GerSTEIN, D. (1987): «To unpack micro and macro», en J. Alexander et al., cit.

Lamo de Espinosa, E., y Rodríguez Ibáñez, J. E. (eds.) (1993): Problemas de teoría social contemporánea, Madrid, Centro de Investigaciones Sociológicas.

MÜNCH, R. (1987): «The interpenetration of microinteraction and macrostructures», en J. Alexander et al., cit.

RODRígueZ IbÁNEZ, J. E. (1991): «Decisión racional versus holismo», REIS, núm. 54.

- (1992): La perspectiva sociológica. Historia, teoría y método, Madrid, Taurus.

- (1997): «Las cuatro "avenidas fuertes" de la teoría sociológica contemporánea, Papers (Barcelona), núm. 50 (enero).

Rumelhart, D. E., y McLelland, J. L. (comps.) (1986): Parallel distributive processing, vols. I y II, Cambridge (Mass.), MIT Press.

SCHEFf, T. (1990): Microsociology, Chicago, University of Chicago Press.

VARELA, F., et al. (1992): De cuerpo presente. Las ciencias cognitivas y la experiencia humana, Barcelona, Gedisa.

* Hallándose ya esta nota en fase de corrección de pruebas, me topo con la valiosa compilación de Manuel Cruz, Acción humana (Barcelona, Ariel, 1997). Me contento con mencionarla, resaltando de su contenido la importante contribución de Salvador GinER, "Intenciones humanas, estructuras sociales», y el ilustrativo resumen de Amparo GÓMEZ, "Microfundamentos de la explicación social». 


\title{
PALABRAS CLAVE
}

- Micro.

- Macro.

- Cognitivismo.

- Emociones.

- Teoría sociológica.

\section{KEYWORDS}

- Micro.

- Macro.

- Cognitive science.

- Emotions.

- Sociological theory.

\begin{abstract}
This paper analyzes the micro-macro link in Sociology from a gradational perspective which tries to avoid dualism on both theoretical and methodological grounds. In doing so, it relies on classic and contemporary models of sociological theory, particularly discussing cognitive and emotions-oriented contributions by Cicourel, Collins and Scheff.
\end{abstract}

